\title{
Aberrational vignetting and eikonal theory
}

\section{Andrey Gitin \\ agitin@mbi-berlin.de}

Max-Born-Institut für Nichtlineare Optik und Kurzzeitspectroskopie, Max-Born-Str. 2a, D-12489 Berlin, Germany

If an aperture stop is located inside of a real optical system, then a variation of an entrance pupil depends on the inclination of the incident beam of light rays. This effect called "aberrational vignetting" depends on aberration of the part of the optical system, which is located between the aperture stop and the entrance pupil. A theory of aberrational vignetting has been developed using the eikonal theory. [DOI: 10.2971/jeos.2009.09046]

Keywords: aberration, apertures, eikonal theory, radiometry, vignetting

\section{INTRODUCTION}

It is well known that there is an unintended fall of the illumination from the center to the edge of the image of the optical systems (such as camera objectives). This is the vignetting phenomena $[1,2]$. The reason of the phenomena is the change of the sizes of homocentric beams of light rays emitting from different points of the source-object. Let us consider the case when the sizes of beams are limited by a single stop, so-called aperture stop.

In the case when the aperture stop is located in object space we deal with natural vignetting (also known as a natural illumination fall-off) [1]-[5] (see Figure 1). This aperture stop is an entrance pupil which subtends a homocentric beam emitted by an object point. By the natural vignetting the angular aperture $\Delta \omega$ of the off-axis homocentric beam is a monotonically decreasing function of distance $x$ between the object point and the optical axis. If the source-object obeys the Lambertian law and the diameter of the aperture stop $D$ is small in comparison with the distance $z$ between an image plane and aperture stop, the natural vignetting is described by the "cosine fourth" law of illumination fall-off [3]-[5]. Natural vignetting is inherent to each lens design and becomes more troublesome for wide angle lenses.

The natural illumination fall-off is not a theoretical inevitability. In 1938 M. M. Rusinov [6, 7] discovered the phenomenon of aberrational vignetting, which made it possible to radically improve the natural illumination fall-off over the field of view of objectives. If the aperture stop were to take place inside of an optical system between two lens groups, it would, with the help of the pupil aberrations, be able to increase light entering the pupil for oblique rays. The growth of the entrance pupil compensates for the natural illumination fall-off.

In the present work we describe the phenomenon of aberrational vignetting using mathematical tools of the eikonal the- ory [8]-[10], as well as the method of energy calculation of optical systems with a planar Lambertian object-source [11, 12].

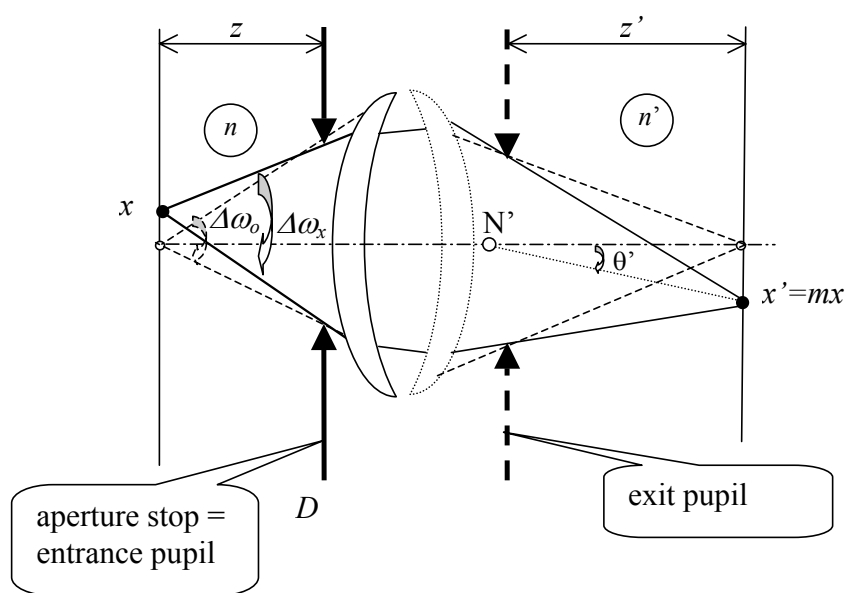

FIG. 1 Natural vignetting.

\section{THEORY}

It is known $[4,8]-[10,13]$ that the application of the eikonal theory to radiometric calculations is simplified by using the concept of phase radiance. The "phase radiance" $L\left(x, y ; p_{x}, p_{y}\right)$ is defined as the distribution of radiation flux $F$ over the phase space $\left(x, y ; p_{x}, p_{y}\right)[10,13]$,

$$
d^{4} F=\frac{L\left(x, y ; p_{x}, p_{y}\right)}{n^{2}} d x d y d p_{x} d p_{y} .
$$

Here $(x, y)$ are the coordinates on the object plane, $\left(p_{x}, p_{y}\right)=\left(n \cdot e_{x}, n \cdot e_{y}\right)$ are the momenta, where $\left(e_{x}, e_{y}\right)$ $=(\sin \theta \cdot \sin \varphi, \sin \theta \cdot \cos \varphi)$ are the directional cosines of the ray in the object space. For Lambertian sources the phase radiance is constant over all "coordinate-momentum" of phase space: $L\left(x, y ; p_{x}, p_{y}\right)=L=$ const. 
Let us consider an axially-symmetrical optical system with a round aperture stop placed inside of the optical system in a plane $\left(x_{0}, y_{0}\right)$. Let the optical system as the whole be aberration-free. The part between the object plane $(x, y)$ and the plane of the aperture stop $\left(x_{0}, y_{0}\right)$ can have aberrations (so-called pupil aberrations), but the other part of this system improves them. Note that in such systems the process of restriction of beams of light rays is convenient for describing in space of subjects.

We now consider two special cases; the object is located at a finite distance (see Figure 2(a)) and the object is located at infinity (see Figure 2(b)).

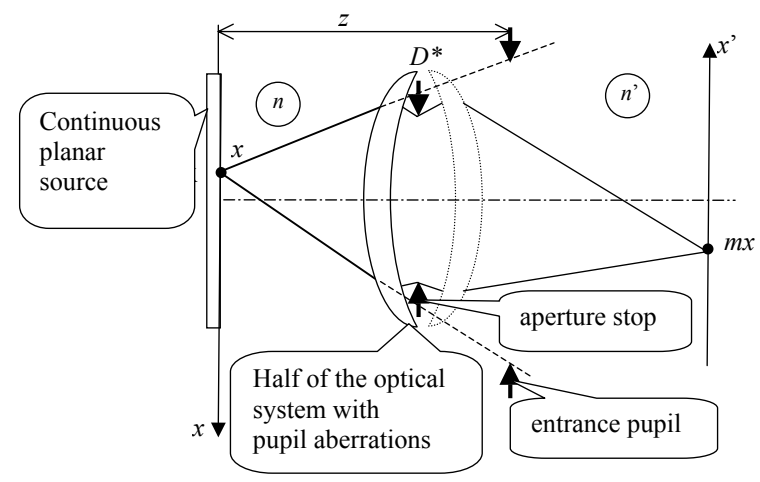

(a)

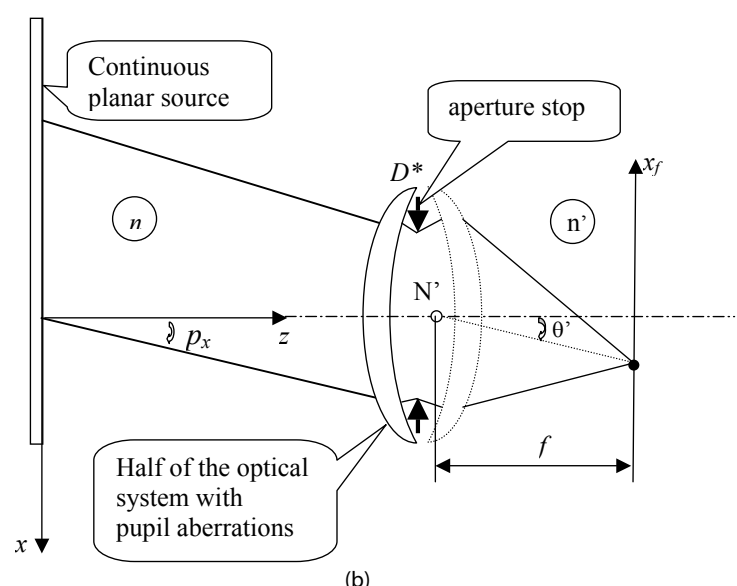

FIG. 2 Aberrational vignetting; (a) the object is located at a finite distance, (b) the object is located at infinity.

\subsection{The object is located at a finite distance}

A homocentric beam of light rays leaves an infinite small surface element $d x d y$ containing the point $(x, y)$ of the planar source-object. After passing an entrance pupil of the optical system it is collected at the optically conjugated infinite small surface element $d x^{\prime} d y^{\prime}$ containing the point $\left(x^{\prime}, y^{\prime}\right)$. In the case of an axially-symmetrical optical system it is possible, without loss of generality, to consider a simple case where this homocentric beam of light rays leaves the point $(x, 0)$ and is collected at the optically conjugated point $\left(x^{\prime}, 0\right)$.

The size of this homocentric beam is limited by a round aperture stop of diameter $D^{*}$, but because of pupil aberrations, this beam borrows in the momentum plane a non-round area. Ac- cording to Rusinov [7] its form is approximately equal to an ellipse with the semi-major axis length $\Delta p_{x}$ and the semi-minor axis length $\Delta p_{y}$, so the area $P$ is equal to

$$
P=\pi \Delta p_{x} \Delta p_{y} \text {. }
$$

To define the semi-axes $\Delta p_{x}$ and $\Delta p_{y}$ of the ellipse in the momenta plane, we shall use the point eikonal $S\left(x, 0, x_{0}, y_{0}\right)$ [8]$[10,13,14]$ provided that the aperture stop is reasonably small: $D^{*}<<z$. In the meridional section the momenta of the light rays connecting the point $(x, 0)$ with the edges of an aperture diaphragm we shall find from expressions

$$
\begin{aligned}
& p_{x}^{+}=\frac{\partial S\left(x, 0 ; D^{*} / 2,0\right)}{\partial x} \\
& p_{x}^{-}=\frac{\partial S\left(x, 0 ;-D^{*} / 2,0\right)}{\partial x} .
\end{aligned}
$$

So in linear approximation we have

$$
\begin{aligned}
2 \Delta p_{x} & =p_{x}^{+}-p_{x}^{-} \\
& =\frac{\partial S\left(x, 0 ; D^{*} / 2,0\right)}{\partial x}-\frac{\partial S\left(x, 0 ;-D^{*} / 2,0\right)}{\partial x} \\
& \approx \frac{\partial^{2} S(0,0 ; 0,0)}{\partial x \partial x_{0}} D^{*} .
\end{aligned}
$$

In the sagittal section we use expressions

$$
\begin{aligned}
& p_{y}^{+}=\frac{\partial S\left(x, 0 ; 0, D^{*} / 2\right)}{\partial y} \\
& p_{y}^{-}=\frac{\partial S\left(x, 0 ; 0,-D^{*} / 2\right)}{\partial y} .
\end{aligned}
$$

So in linear approximation we have

$$
\begin{aligned}
2 \Delta p_{y} & =p_{y}^{+}-p_{y}^{-} \\
& =\frac{\partial S\left(x, 0 ; 0, D^{*} / 2\right)}{\partial y}-\frac{\partial S\left(x, 0 ; 0,-D^{*} / 2\right)}{\partial y} \\
& \approx \frac{\partial^{2} S(0,0 ; 0,0)}{\partial y \partial y_{0}} D^{*} .
\end{aligned}
$$

Thus the substitution of Eqs. (3) and (4) into Eq. (2) yields

$$
\begin{aligned}
P & \equiv \pi \Delta p_{x} \Delta p_{y} \\
& \approx \frac{\pi}{4} \frac{\partial^{2} S(x, 0 ; 0,0)}{\partial x \partial x_{0}} \frac{\partial^{2} S(x, 0 ; 0,0)}{\partial y \partial y_{0}} D^{* 2} .
\end{aligned}
$$

In the case of a planar Lambertian source according to Eq. (1) the flux $d^{2} F$ emitted by a surface element $d x d y$ containing the point $(x, 0)$ and restricted by the aperture stop $P$ is equal to

$$
d^{2} F=\frac{L}{n^{2}} d x d y \cdot P .
$$

This flux $d^{2} F$ is collected without losses at the optically conjugate surface element $d x^{\prime} d y^{\prime}$ of the image of the perfect optical system: $d x^{\prime} d y^{\prime}=m^{2} d x d y$, where $m$ is its lineal magnification. Thus, the required illumination distribution fall-off from the centre to the edge of the image plane $E\left(x^{\prime}, 0\right)$ can be found from

$$
E\left(x^{\prime}, 0\right) \equiv \frac{d^{2} F}{d x^{\prime} d y^{\prime}}=\frac{L}{(n m)^{2}} \cdot P .
$$

Substitution of Eq. (5) into Eq. (7) yields

$$
E\left(x^{\prime}, 0\right)=\frac{\pi L}{4(n m)^{2}} \frac{\partial^{2} S\left(x^{\prime} / m, 0 ; 0,0\right)}{\partial x \partial x_{0}} \frac{\partial^{2} S\left(x^{\prime} / m, 0 ; 0,0\right)}{\partial y \partial y_{0}} D^{* 2} .
$$


In the particular case of the "natural vignetting", when a reasonably small aperture stop (or its perfect image) is located in the object space (and carries out the function of an entrance pupil), the point eikonal is a point eikonal of the layer of optically homogeneous media with refraction index $n$ and thickness $z$ (see Figure 1),

$$
S\left(x, y ; x_{0}, y_{0}\right)=\left.n \sqrt{z^{2}+\left(x_{o}-x\right)^{2}+\left(y_{0}-y\right)^{2}}\right|_{y=0} .
$$

Thus Eq. (8) becomes:

$$
E^{o}\left(x^{\prime}, 0\right)=\frac{\pi L}{4}\left(\frac{D}{m z}\right)^{2} \frac{1}{\left[1+\left(x^{\prime} / m z\right)^{2}\right]^{2}}
$$

where $D$ is the diameter of the entrance pupil.

According to Maxwell's formula [11] $m \cdot m_{0} \cdot z=\left(n / n^{\prime}\right) z^{\prime}$, where $n$ and $n^{\prime}$ are indices of refraction in the object and image spaces respectively, $z^{\prime}$ is the distance between the exit pupil and image plane, $m_{0}$ is a linear magnification in the pupil planes. So, Eq. (9) can be rewritten in the following way:

$$
E^{o}\left(x^{\prime}, 0\right)=\frac{\pi L}{4}\left(\frac{n^{\prime} m_{o}}{n z^{\prime}} D\right)^{2} \frac{1}{\left[1+\left(n^{\prime} m_{o} x^{\prime} / n z^{\prime}\right)^{2}\right]^{2}} .
$$

In the typical case of $n=n^{\prime}=1$ and $m_{o}=1$ the Eq. (10) takes the form of the famous "cosine fourth" law:

$$
E^{o}\left(x^{\prime}, 0\right)=\frac{\pi L}{4}\left(\frac{D}{z^{\prime}}\right)^{2} \cos ^{4} \theta^{\prime}\left(\frac{x^{\prime}}{z^{\prime}}\right) .
$$

Here $\cos \theta^{\prime}\left(x^{\prime} / z^{\prime}\right)=1 / \sqrt{1+\left(x^{\prime} / z^{\prime}\right)^{2}}$ and $\theta^{\prime}$ is the angle in an image space counted from the back nodal point $N^{\prime}$ (see Figure 1). Note that the "natural vignetting" Eq. (10) enables us to rewrite Eq. (8) in the form of the product

$$
E\left(x^{\prime}, 0\right)=E^{o}\left(x^{\prime}, 0\right) \mu\left(x^{\prime}, 0\right)
$$

where

$$
\begin{aligned}
\mu\left(x^{\prime}, 0\right)=\left(\frac{z^{\prime}}{m_{0} n^{\prime}}\right)^{2}\left[1+\left(\frac{n^{\prime} m_{o}}{n z^{\prime}} x^{\prime}\right)^{2}\right]^{2} \\
\quad \times \frac{\partial^{2} S\left(x^{\prime} / m, 0 ; 0,0\right)}{\partial x \partial x_{0}} \frac{\partial^{2} S\left(x^{\prime} / m, 0 ; 0,0\right)}{\partial y \partial y_{o}}
\end{aligned}
$$

is a factor describing aberrational vignetting of the optical system. In the first approximation the factor does not depend on the diameter of the aperture stop [7]. Thus, in the case when the object-source is located at a finite distance, aberrational vignetting depends on the product of mixed derivatives of the point eikonal $S$ in meridional and sagittal planes.

\subsection{The object is located at infinity}

If the planar object-source is moved to infinity: $z \rightarrow \infty$, then $m \rightarrow 0$ and the image plane transforms in the back focal plane: $\left(x^{\prime}, y^{\prime}\right) \rightarrow\left(x_{f}, y_{f}\right)$, where $f$ is the focal length, i.e. the distance between the back focal point and the back nodal point $N^{\prime}$ (see Figure 2(b)).

A parallel beam of light rays with the momenta $\left(p_{x}, p_{y}\right)$ and a small divergence $d p_{x} d p_{y}$ leaves the planar object-source and falls to the entrance pupil of the optical system. After the beam passes the entrance pupil, it is collected at an infinite small surface element of the back focal plane

$$
d x_{f} d y_{f}=f^{2} d p_{x} d p_{y}
$$

containing the point $\left(x_{f}, y_{f}\right)=\left(f \cdot p_{x}, f \cdot p_{y}\right)$. In the case of an axially-symmetrical optical system it is possible, without loss of a generality, to consider a simpler case in which this parallel beam of rays with the momenta $\left(p_{x}, 0\right)$ is focused into the point $\left(x_{f}, 0\right)$, where

$$
p_{x}=\frac{n \cdot x_{f}}{\sqrt{f^{2}+x_{f}^{2}}} .
$$

The size of this beam is limited by a round aperture stop of diameter $D^{*}$ but, because of pupil aberration, this beam borrows in the coordinate plane a non-round area. According to Rusinov [7] its form is approximately equal to an ellipse with semi-major axis length $\Delta x$ and semi-minor axis length $\Delta y$, so the area $P$ is equal to

$$
Q=\pi \Delta x \Delta y
$$

To define the semi-axes $\Delta x$ and $\Delta y$ of the ellipse in the coordinate plane we use the angle-point eikonal $V^{\prime}\left(p_{x}, 0, x_{0}, y_{0}\right)$ [14]. In the meridional section the coordinates of the border of the entrance pupil can be found from the expressions

$$
\begin{aligned}
& x^{+}=\frac{\partial V^{\prime}\left(p_{x}, 0 ; D^{*} / 2,0\right)}{\partial p_{x}} \\
& x^{-}=\frac{\partial V^{\prime}\left(p_{x}, 0 ;-D^{*} / 2,0\right)}{\partial p_{x}} .
\end{aligned}
$$

So in linear approximation we have

$$
\begin{aligned}
2 \Delta x & =x^{+}-x^{-} \\
& =\frac{\partial V^{\prime}\left(p_{x}, 0 ; D^{*} / 2,0\right)}{\partial p_{x}}-\frac{\partial V^{\prime}\left(p_{x}, 0 ;-D^{*} / 2,0\right)}{\partial p_{x}} \\
& \approx \frac{\partial^{2} V^{\prime}\left(p_{x}, 0 ; 0,0\right)}{\partial p_{x} \partial x_{0}} D^{*} .
\end{aligned}
$$

In the sagittal section we use the expressions

$$
\begin{aligned}
& y^{+}=\frac{\partial V^{\prime}\left(p_{x}, 0 ; 0, D^{*} / 2\right)}{\partial p_{y}} \\
& y^{-}=\frac{\partial V^{\prime}\left(p_{x}, 0 ; 0,-D^{*} / 2\right)}{\partial p_{y}} .
\end{aligned}
$$

So in linear approximation we have

$$
\begin{aligned}
2 \Delta y & =y^{+}-y^{-} \\
& =\frac{\partial V^{\prime}\left(p_{x}, 0 ; 0, D^{*} / 2\right)}{\partial p_{y}}-\frac{\partial V^{\prime}\left(p_{x}, 0 ; 0,-D^{*} / 2\right)}{\partial p_{y}} \\
& \approx \frac{\partial^{2} V^{\prime}\left(p_{x}, 0 ; 0,0\right)}{\partial p_{y} \partial y_{0}} D^{*} .
\end{aligned}
$$

Thus the substitution of Eqs. (16) and (17) into Eq. (15) yields

$$
Q=\frac{\pi}{4} \frac{\partial^{2} V^{\prime}\left(p_{x}, 0 ; 0,0\right)}{\partial p_{x} \partial x_{o}} \frac{\partial^{2} V^{\prime}\left(p_{x}, 0 ; 0,0\right)}{\partial p_{y} \partial y_{o}} D^{* 2} .
$$

Let us consider a parallel beam of light rays with the momenta $\left(p_{x}, 0\right)$ and a small divergence $d p_{x} d p_{y}$ that is restricted by the 
entrance pupil $Q$. In the case of a planar Lambertian source according to Eq. (1) the flux $d^{2} F$ carried by this parallel beam is equal to

$$
d^{2} F=\frac{L}{n^{2}} d p_{x} d p_{y} \cdot Q .
$$

This flux $d^{2} F$ is collected without losses at the surface element $d x_{f} d y_{f}$ of the back focal plane. The required illumination distribution fall-off from the centre to the edge of the back focal plane $E\left(x_{f}, 0\right)$ is proportional to the area $Q$ of the entrance pupil and can be calculated by the formula [11, 12],

$$
E\left(x_{f}, 0\right)=\frac{L}{f^{2}} Q \cos ^{4} \theta^{\prime}\left(x_{f}, 0\right) \text {. }
$$

Here $\cos \theta^{\prime}\left(x_{f}, 0\right)=1 / \sqrt{1+\left(x_{f} / f\right)^{2}}$, where $\theta^{\prime}$ is an angle in image space counted from the back nodal point $N^{\prime}$ (see Figure 2(b)). Substitution of Eqs. (18) and (14) into Eq. (20) yields

$$
\begin{aligned}
& E\left(x_{f}, 0\right)=\frac{\pi}{4} \frac{L}{f^{2}} \frac{\partial^{2} V^{\prime}\left(n x_{f} / \sqrt{f^{2}+x_{f}^{2}}, 0 ; 0,0\right)}{\partial p_{x} \partial x_{o}} \\
& \times \frac{\partial^{2} V^{\prime}\left(n x_{f} / \sqrt{f^{2}+x_{f}^{2}}, 0 ; 0,0\right)}{\partial p_{y} \partial y_{o}} D^{* 2} \frac{1}{\left[1+\left(x_{f} / f\right)^{2}\right]^{2}} .
\end{aligned}
$$

In the case of "natural vignetting", when the aperture stop is located in the object space (and carries out the functions of an entrance pupil), the Eq. (21) becomes [11, 12],

$$
E\left(x_{f}, 0\right)=\frac{\pi}{4} \frac{L}{f^{2}} D^{* 2} \frac{1}{\left[1+\left(x_{f} / f\right)^{2}\right]^{2}} .
$$

Note that the "natural vignetting" Eq. (22) enables us to rewrite Eq. (21) in the form of the product

$$
E\left(x_{f}, 0\right)=E^{o}\left(x_{f}, 0\right) \mu_{\infty}\left(x_{f}, 0\right)
$$

where

$$
\begin{aligned}
\mu_{\infty}\left(x_{f}, 0\right)=\frac{\partial^{2} V^{\prime}\left(n x_{f} / \sqrt{f^{2}+x_{f}^{2}}, 0 ; 0,0\right)}{\partial p_{x} \partial x_{o}} \\
\times \frac{\partial^{2} V^{\prime}\left(n x_{f} / \sqrt{f^{2}+x_{f}^{2}}, 0 ; 0,0\right)}{\partial p_{y} \partial y_{o}}
\end{aligned}
$$

is a factor describing aberrational vignetting of the optical system. In the first approximation the factor does not depend on the diameter of the aperture stop [7]. Thus, in the case of an object-source located at infinity, the aberrational vignetting depends on a product of mixed derivatives of the angle-point eikonal $\mathrm{V}^{\prime}$ in meridional and sagittal planes.

\section{CONCLUSION}

Aberrational vignetting is achieved by the introduction of aberrations in the part of the optical system located between the aperture stop and the object space.

If the object is located at a finite distance, the efficiency of the aberrational vignetting depends on the mixed derivatives of the point eikonal.

If the object is located at infinity, the efficiency of the aberrational vignetting depends on the mixed derivatives of the angle-point eikonal.

\section{References}

[1] www.vanwalree.com/optics/vignetting.html

[2] www.gimpguru.org/Tutorials/Vignetting/

[3] S. F. Ray, Applied photographic optics, 3rd ed. (Focal Press, 0xford, 2002).

[4] A. V. Gitin, "The Legendre transformation as a way of constructing a phase portrait of beams of light rays" Opt. Commun. 282, 757 (2009).

[5] A. V. Gitin, Radiometry of optical systems with quasi-homogeneous sources: a linear systems approach (4th EOS Topical Meeting on Advanced Imaging Techniques, Jena, Germany, June 10-12, 2009).

[6] “Mikhail Mikhailovich Rusinov (2/11/1909 - 9/30/2004)" J. Opt. Technol.+ 71, 859 (2004).

[7] M. M. Rusinov, Technical Optics (Mashinostroenie, Leningrad, 1979, in Russian).

[8] I. N. Tarnakin, "Determination of illumination and light flux by an optical-system eikonal" Opt. Spectrosc.+ 44, 463 (1978).

[9] A. V. Gitin, "Calculation of the illuminance distribution in the focal spot of a focusing system taking into account aberrations in this system and divergence of a focused laser beam" Quantum Electron.+ 37, 307 (2007).

[10] A. V. Gitin, Aberrational vignetting and eikonal theory (4th EOS Topical Meeting on Advanced Imaging Techniques., Jena, Germany, June 10-12, 2009).

[11] A. V. Gitin, "Radiometry as a section of optical system theory" Opt. Spectrosc.+ 63, 106 (1987).

[12] A. V. Gitin, "The effective point source" Opt. Spectrosc.+ 76, 157 (1994).

[13] A. V. Gitin, "Radiometry. A complex approach" J. Opt. Technol.+ 65, 132 (1998).

[14] M. Born, and E. Wolf, Principles of Optics (Pergamon Press Inc., New York, 1964). 